\title{
Fall detection framework for smart home
}

\begin{abstract}
This paper will describe the concept of fall detection framework for smart home environment which will focus on elderly people. We also discuss and compared general fall detection system and fall detection framework that been implemented. The study of this paper will also help to get understanding about indoor fall detection techniques, advantages, drawbacks and the challenges to enhance near in the future.
\end{abstract}

Keyword: Fall detection framework; Activity recognition; Machine learning language; Fall monitoring; Fall prediction 